\title{
Optical spectroscopy of rare earth-doped oxyfluoro-tellurite glasses to probe local environment
}

\author{
GAJANAN V HONNAVAR ${ }^{1,2, *}$ and K P RAMESH ${ }^{2}$ \\ ${ }^{1}$ PES Institute of Technology, Bangalore South Campus, Near Electronic City, Bangalore 560100, India \\ ${ }^{2}$ Department of Physics, Indian Institute of Science, Bangalore 560012, India \\ *Author for correspondence (gajanan.honnavar@gmail.com)
}

MS received 14 September 2016; accepted 30 November 2016; published online 16 August 2017

\begin{abstract}
TeO}_{2}$-based glasses with a general formula $65 \mathrm{TeO}_{2}-5 \mathrm{BaF}_{2}-30 \mathrm{ZnF}_{2}(\mathrm{TBZ})$ (in mol\%) were prepared by usual melt quenching technique. Three mol\% of europium (Eu) or erbium $(\mathrm{Er})$ were added to the prepared glass at the expense of $\mathrm{TeO}_{2}$. Raman, photoluminescence (PL), UV-visible absorption studies were carried out on the glass samples. Raman spectra of the undoped and doped glasses were analysed using the peak shift and the intensity variation along with full width at half-maximum (FWHM). It was found that Eu-doped TBZ glass has a greater tendency towards depolymerizing the glass matrix by influencing the conversion of $\mathrm{TeO}_{4}$ to $\mathrm{TeO}_{3}$ units compared to Er-doped and undoped glasses. PL spectra of the glass samples show emission due to different possible transitions. Position of the peak of the de-convoluted spectra shows the position of the particular Stark component and the FWHM is a measure of the inhomogeneous broadening. The UV-visible absorption spectra are used to calculate the optical density and to determine the band edge of the glass samples by fitting to the Mott equation. It is seen that Eu-doped TBZ glass has a lesser bandgap than that of Er-doped glass.
\end{abstract}

Keywords. Tellurite glasses; Raman spectroscopy; photoluminscence; Stark level splitting; UV visible spectroscopy.

\section{Introduction}

Rare earth (RE) elements are of special interest in several high-technology and environmental applications, especially in optical and magnetic devices [1]. Optical properties of the RE elements are due to the atomic-like sharp $f-f$ transitions. Even though, these transitions are shielded from the surrounding environment by the outer shell $5 s$ and $5 p$ electrons, the transition probabilities are sensitive to the ions surrounding the RE. Thus, one can get a fair knowledge of the surrounding environment by studying the transitions of RE ions. Judd-Ofelt theory helps in estimating the effect of the surrounding environment on $4 f$ transition probabilities by calculating some intensity parameters $\left(\Omega_{2}, \Omega_{4}, \Omega_{6}\right)[2,3]$. However, such information is limited with respect to the estimation of fine changes in the surrounding environment [4]. Local probes like NMR [5] and Raman can reveal such fine changes in the environment much more effectively.

RE-doped tellurite glasses are widely used as materials for optical fibres. Tellurium dioxide $\left(\mathrm{TeO}_{2}\right)$-based glasses have higher phonon energy $\left(780 \mathrm{~cm}^{-1}\right)$ and refractive index $(>1.9)$ than ZBLAN $\left(\mathrm{ZrF}_{4}-\mathrm{BaF}_{2}-\mathrm{LaF}_{3}-\mathrm{AlF}_{3}-\mathrm{NaF}\right)$ glasses [6]. Erbium (Er)-doped glasses are in use as erbium-doped fibre amplifier (EDFA). Europium (Eu) ions show a strong emission near red region when excited with ultraviolet and can be used as an ultraviolet detector. Optical properties of RE ion may show pronounced behaviour depending on the variation of host glass matrix. Hence, structural characterization of a glass matrix with different RE ions for optical properties is of importance.

Oxide glasses have good chemical durability, but low glassforming ability and their melting temperature is usually high. All fluoride glasses have low-melting temperature, but their chemical durability is weak $[7,8]$. Oxyfluoride glasses are characterized with extensive composition, high content of doped RE ions, low non-linear refractive index and high Abbe index [9].

Keeping this in view, we have chosen oxyfluoro-tellurite glass matrix and investigated the structural aspects using optical spectroscopic techniques like Raman, photoluminescence (PL) and UV-visible absorption on the RE (Eu and Er) iondoped oxyfluro-tellurite glasses.

\section{Experimental}

\subsection{Sample preparation and basic characterization}

$\mathrm{TeO}_{2}$-based glasses with a general formula $65 \mathrm{TeO}_{2}-5 \mathrm{BaF}_{2}-$ $30 \mathrm{ZnF}_{2}$ (TBZ) (in mol\%) were prepared by usual melt quenching technique. RE doping was done at the expense of $\mathrm{TeO}_{2}$ with $3 \mathrm{~mol} \%$ of Eu or Er in the above glass matrix. Calculated amount of the starting materials $\left(\mathrm{TeO}_{2}, \mathrm{BaF}_{2}, \mathrm{ZnF}_{2}\right.$ and $\mathrm{Eu}_{2} \mathrm{O}_{3}$ or $\mathrm{Er}_{2} \mathrm{O}_{3}$; all from Sigma Aldrich) were taken in a platinum crucible; mixed thoroughly and heated around $800^{\circ} \mathrm{C}$ for $20 \mathrm{~min}$ and quenched between two brass plates. 


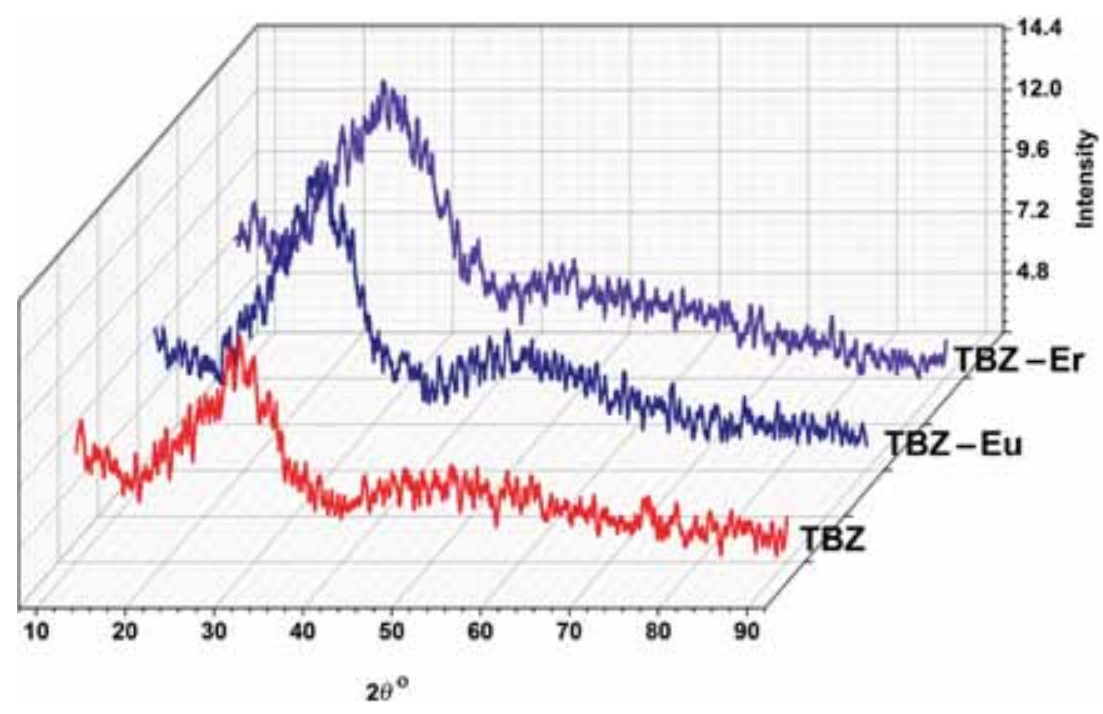

Figure 1. XRD pattern of the tellurite glasses studied.

Table 1. Composition and glass transition temperature, $T_{\mathrm{g}}$ of TBZ glasses.

\begin{tabular}{lcccccc}
\hline \multirow{2}{*}{$\begin{array}{l}\text { Glass } \\
\text { code }\end{array}$} & \multicolumn{5}{c}{ Content of the glass (mol\%) } & Glass transition \\
\cline { 2 - 5 } & $\mathrm{TeO}_{2}$ & $\mathrm{BaF}_{2}$ & $\mathrm{ZnF}_{2}$ & $\mathrm{Eu}_{2} \mathrm{O}_{3}$ & $\mathrm{Er}_{2} \mathrm{O}_{3}$ & \\
temperature, $T_{\mathrm{g}},{ }^{\circ} \mathrm{C}\left(0.05^{\circ} \mathrm{C}\right)$ \\
\hline $\mathrm{TBZ}$ & 65 & 5 & 30 & - & - & 337.69 \\
$\mathrm{TBZ}-\mathrm{Eu}$ & 62 & 5 & 30 & 3 & - & 344.28 \\
$\mathrm{TBZ}-\mathrm{Er}$ & 62 & 5 & 30 & - & 3 & 361.55 \\
\hline
\end{tabular}

The glasses were transparent with a tinge of greenish colour with no phase separation. The glass transition temperature $T_{\mathrm{g}}$ was determined using differential scanning calorimetry (DSC). The glass samples were annealed at $30^{\circ} \mathrm{C}$ below $T_{\mathrm{g}}$. $\mathrm{X}$-ray diffraction (XRD) (see figure 1) was performed on finely powdered glass samples to check the amorphous nature. Details of composition, nomenclature and $T_{\mathrm{g}}$ are summarized in table 1 .

\subsection{Raman, PL and UV-visible absorption spectroscopy}

Room temperature micro-Raman studies on these samples were carried out in back scattering geometry using HORIBA JOBIN YVON LabRAM HR instrument equipped with a charge-coupled device detector (CCD) at a working temperature of $-70^{\circ} \mathrm{C}$. The samples were illuminated by $325 \mathrm{~nm}$ line of a He-Cd laser focussed, using a 100X NUV objective on thin glass samples. Experimental data were recorded using $<3 \mathrm{~mW}$ of laser power (at the laser head) and for $10 \mathrm{~s}$ acquisition (per window) time with a spectral resolution of $0.4 \mathrm{~cm}^{-1}$. The laser intensity was kept low, so as to avoid damage of the samples. The Raman peaks corresponding to $\mathrm{Te}-\mathrm{O}$ bonds in different network-forming units (discussed below) are deconvoluted using Gaussian line shape. Parameters like peak shift and FWHM were derived from the fit. Error bars on

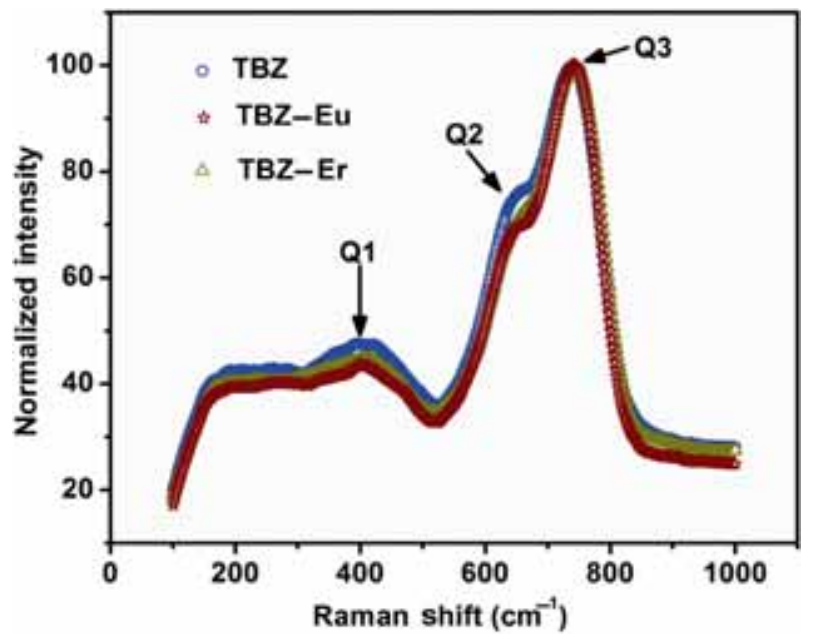

Figure 2. Intensity-normalized Raman spectra showing Q1, Q2 and Q3 bands. The details of the band assignment are discussed in text.

these plots show the error involved in the measured parameters. The usefulness of these parameters in determining the local structure of the samples are discussed in the next section. The same instrument used to record Raman data, was used for PL studies also in the PL mode. PL spectra were 


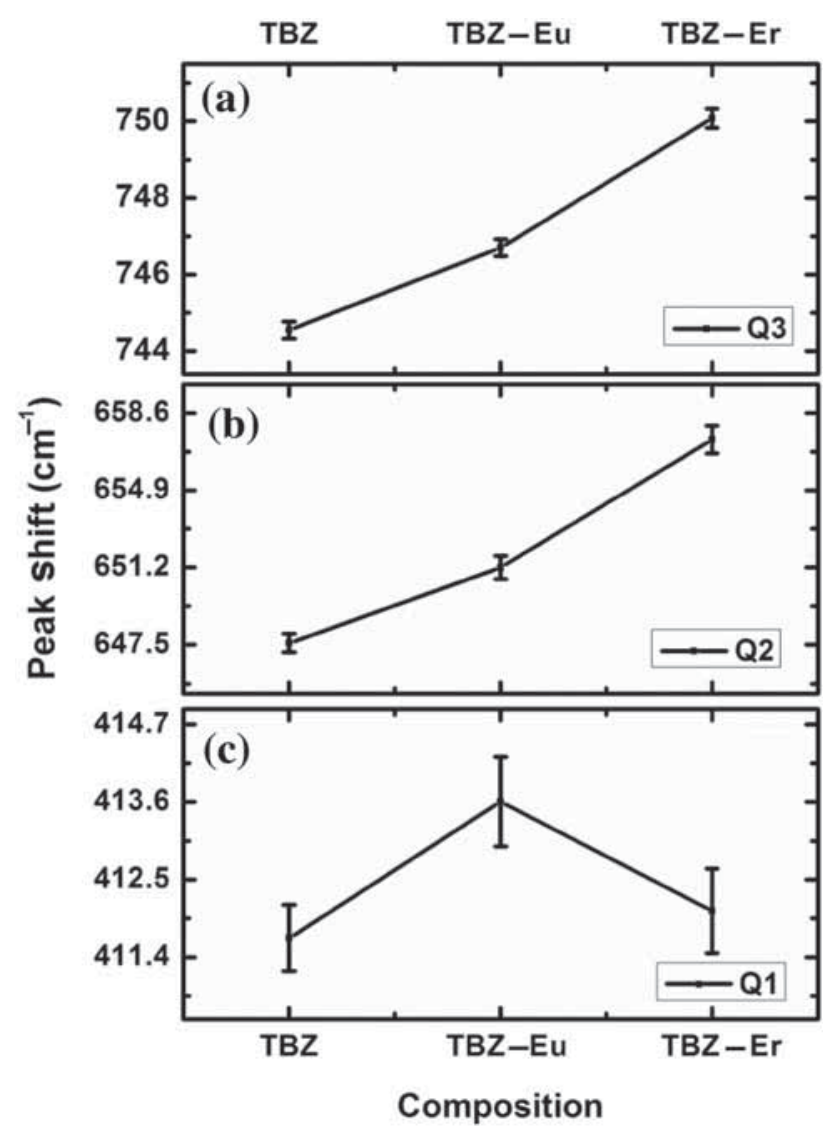

Figure 3. (a-c) Raman peak shift vs. glass composition of Q3, Q2 and Q1 bands, respectively.

recorded at room temperature from 400 to $800 \mathrm{~nm}$ with $\mathrm{He}-\mathrm{Cd}$ laser working at $325 \mathrm{~nm}$ wavelength as the excitation source. The resolution of the spectrometer was $0.014 \mathrm{~nm}$. UV-visible absorption spectra were recorded from 200 to $800 \mathrm{~nm}$ using Cintra $40 \mathrm{UV}-$ Vis spectrometer with $200 \mathrm{~nm} \mathrm{~min}^{-1}$ and $1 \mathrm{~nm}$ resolution.

\section{Results and discussion}

\subsection{Raman spectroscopy}

The tellurite glasses contain three network-forming units viz., $\mathrm{TeO}_{4}$ trigonal bipyramidal (tbp) units, $\mathrm{TeO}_{3}$ trigonal pyramids (tp) and an intermediate unit $\mathrm{TeO}_{3+1}$, which resembles $\mathrm{TeO}_{4}$, but one axial Te-O bond is larger. Figure 2 shows intensitynormalized Raman spectra of all the samples and the position of Q1, Q2 and Q3 bands, which were assigned following Sekiya et al [10]. Q1 band (centred around $400 \mathrm{~cm}^{-1}$ ) corresponds to bending mode of $\mathrm{Te}-\mathrm{O}-\mathrm{Te}$, which is formed by sharing vertices of $\mathrm{TeO}_{4}$ tbp's, $\mathrm{TeO}_{3+1}$ polyhedra and $\mathrm{TeO}_{3}$ tp's; stretching over long distance in the glass network [10]. The band Q2 (centred around $650 \mathrm{~cm}^{-1}$ ) corresponds to symmetric stretching of $\mathrm{Te}-\mathrm{O}$ bonds in $\mathrm{TeO}_{4}$ units and

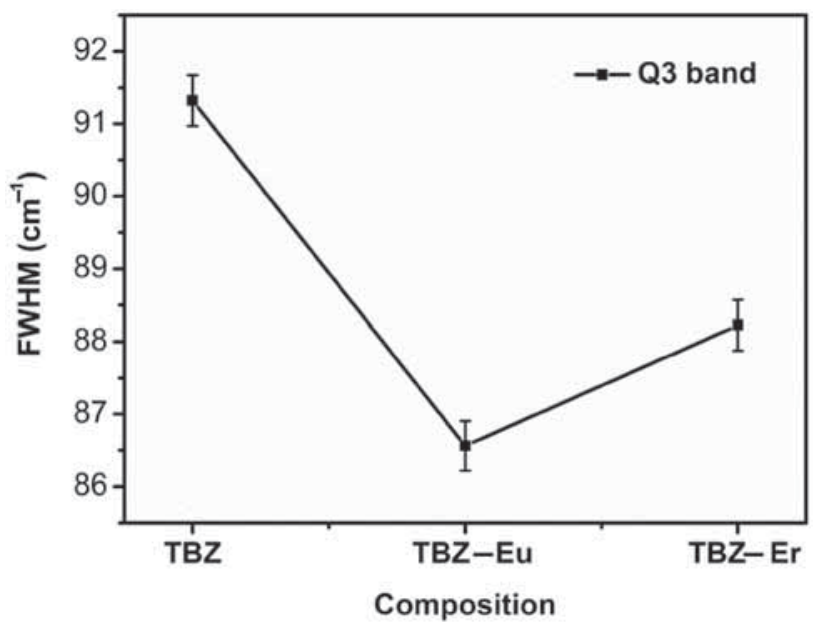

Figure 4. FWHM of $\mathrm{Q} 3$ band corresponding to $\mathrm{TeO}_{3}$ and $\mathrm{TeO}_{3+1}$ groups.
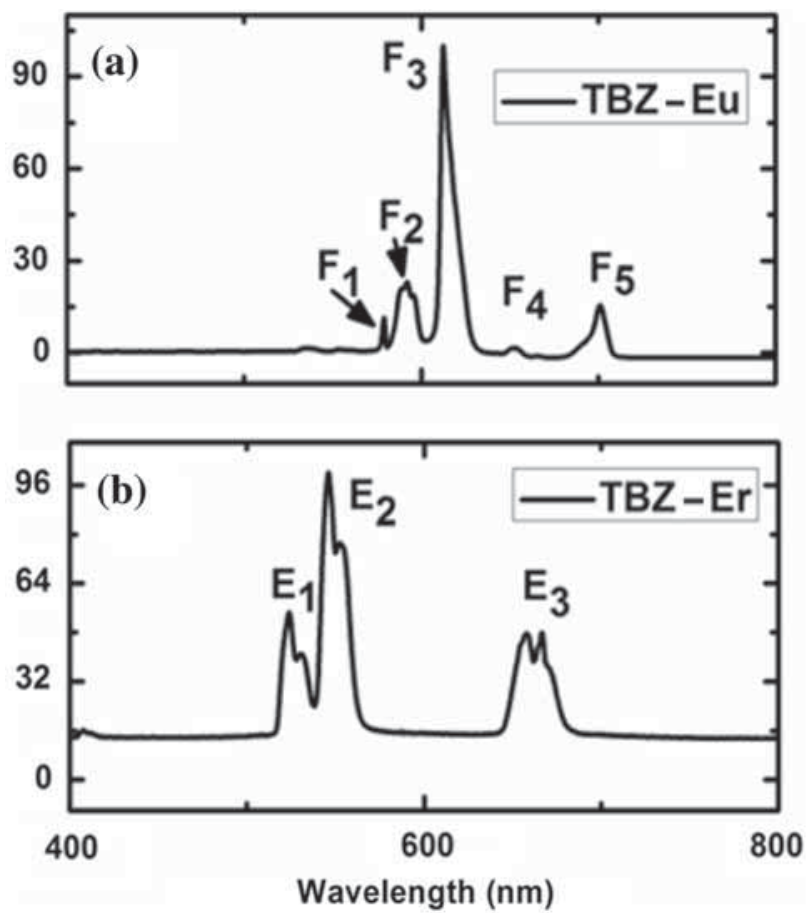

Figure 5. (a, b) Photoluminescence spectra of Eu-doped and Erdoped TBZ glasses, respectively. $Y$-axis represents normalized intensity in arbitrary units.

the Q3 (centred around $750 \mathrm{~cm}^{-1}$ ) is due to Te-O symmetric stretch of $\mathrm{TeO}_{3}$ units. It also includes Te-O stretching mode from an intermediate state $\mathrm{TeO}_{3+1}$, which represents change from $\mathrm{TeO}_{4}$ to $\mathrm{TeO}_{3}$. Raman intensity of a vibrational mode can be related to phonon density of that particular mode [11]. From figure 2, we observe that intensity of Q1 band decreases gradually from undoped TBZ glass to doped glasses reaching minimum at Eu-doped glass. The decrease in intensity is an indication that the long chains containing 

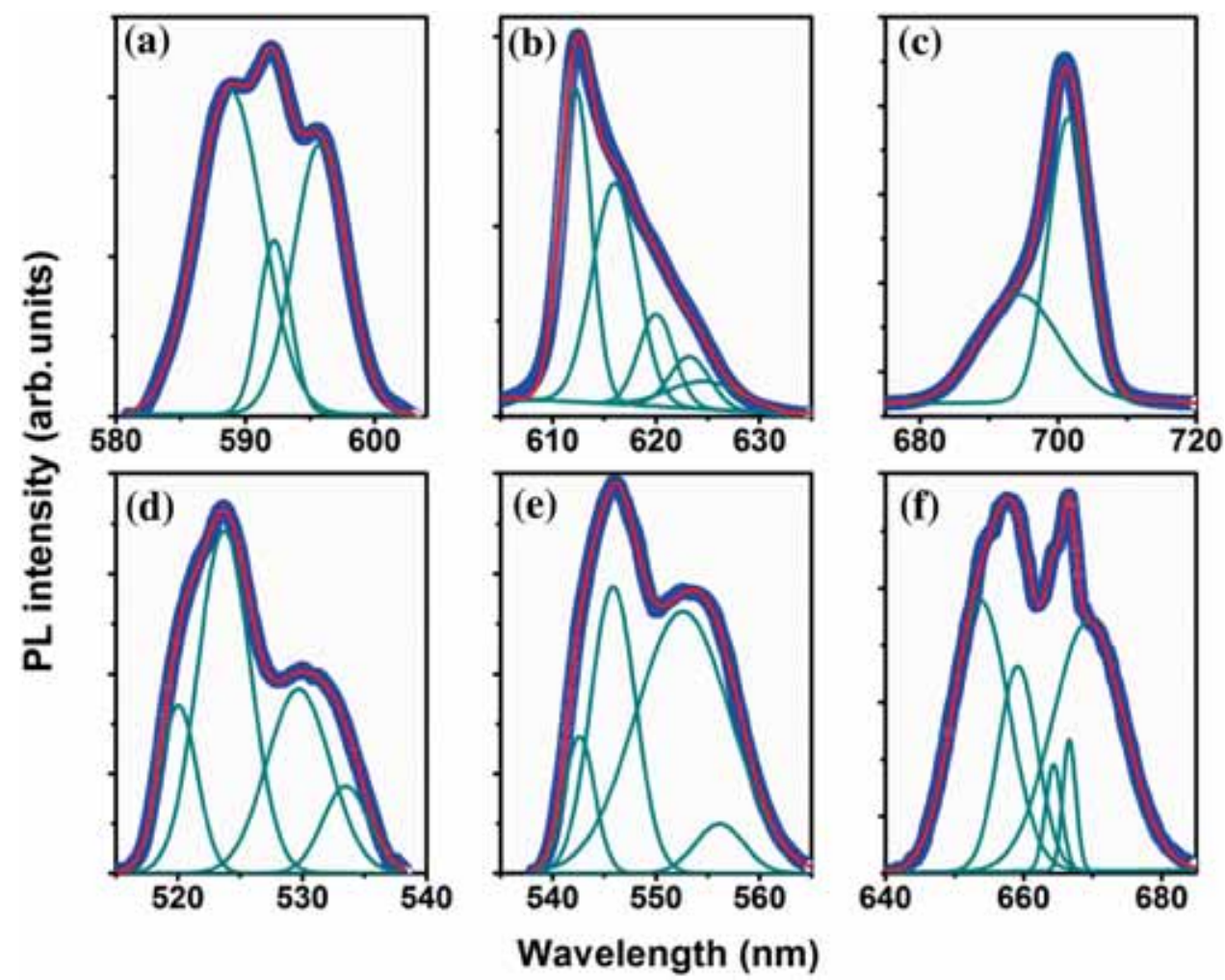

Figure 6. De-convoluted photoluminescence spectra of Eu- and Er-doped TBZ glasses showing Stark splitting. (a-c) Correspond to $F_{2}, F_{3}$ and $F_{5}$ transitions of Eu-doped TBZ glass in figure 5a. (d-e) Correspond to $E_{1}, E_{2}$ and $E_{3}$ transitions of Er-doped glasses in figure 5b. The number of Gaussian profiles fitted to a particular transition represents the extent of Stark splitting.

Te-O-Te over the glass network become increasingly depolymerized as $\mathrm{TeO}_{4}$ units are getting converted to $\mathrm{TeO}_{3}$ units [12].

Figure 3 shows Raman peak shift of the three bands with respect to composition. Figure $3 \mathrm{a}$ and $\mathrm{b}$ shows the peak shift of Q3 and Q2 bands with glass composition. We observe that the behaviour of Q2 and Q3 are identical with the composition, but the extent of shift in Q2 band is greater than Q3 band for a given RE element. Both figures show that shift is larger in Er-doped TBZ glasses than the Eu-doped one with respect to undoped TBZ glass. Figure $3 \mathrm{c}$ shows the variation of Q1 band with the glass composition. The value of Q1 band shift is large for Eu-doped TBZ glass than Er-doped glass. By introducing network modifiers such as RE ions, long Te-O-Te chains get interrupted and this depolymerization brings a shift in the observed Raman spectra. This can be attributed to the ionic radii of the RE ions [12]. $\mathrm{Eu}^{3+}$ is larger with ionic radius $\sim 108.7 \mathrm{pm}$, whereas $\mathrm{Er}^{3+}$ is $103 \mathrm{pm}$. A larger ion can break the chain and readily depolymerize the network [9]. This observation is consistent with the analysis made on the intensity variation in the previous paragraph.

Glasses inherently have a non-periodic nature with random distribution of network-forming units and network modifiers. Because of this irregular arrangement of network units in TBZ glasses, the $\mathrm{TeO}_{4}$ and $\mathrm{TeO}_{3}$ polyhedra will have a distribution of $\mathrm{Te}-\mathrm{O}$ bond lengths around a most probable value. The width of Raman spectral peaks (FWHM) can be attributed to the distribution in a particular bond length [13]. From figure 4, we observe that the distribution of bond length is least for Q3 band in Eu-doped TBZ glass $\left(\sim 5 \mathrm{~cm}^{-1}\right)$ as opposed to Er-doped glass $\left(\sim 3 \mathrm{~cm}^{-1}\right)$ with respect to undoped TBZ glass. This variation of FWHM indicates that Eu-doped glass has a more homogeneous distribution of $\mathrm{Te}-\mathrm{O}$ bond corresponding to $\mathrm{TeO}_{3}$ units than Er-doped glass. Q3 band corresponds to symmetric stretching of $\mathrm{Te}-\mathrm{O}$ bond in both $\mathrm{TeO}_{3+1}$ and $\mathrm{TeO}_{3}$ units. As one of the $\mathrm{Te}-\mathrm{O}$ bond is longer in $\mathrm{TeO}_{3+1}$ unit; it should have given rise to a larger broadening of the $\mathrm{Q} 3$ band. But the absence of such broadening of the corresponding peak indicates that $\mathrm{TeO}_{3}$ units are more than the $\mathrm{TeO}_{3+1}$ units in Eu-doped TBZ glass. This observation again indicates that the tendency to convert $\mathrm{TeO}_{4}$ units into $\mathrm{TeO}_{3}$ units is higher in Eu-doped TBZ glass.

\subsection{Photoluminescence}

Figure 5 shows room temperature PL spectra of the RE-doped glasses from 400 to $800 \mathrm{~nm}$. The intensities of these plots are normalized to the maximum emission line. PL spectrum of Eu-doped TBZ glass is shown in figure 5a. Five emission 
bands viz., $F_{1}-F_{5}$ represent the transitions from the only fluorescent ${ }^{5} D_{0} \rightarrow{ }^{7} F_{n}$ levels, where $n$ corresponds to different total spin levels from 0 to 4 [14]. The other two transitions corresponding to total spin levels 5 and 6 are not observed. Figure 5b shows the PL spectrum of Er-doped TBZ glass. This spectrum is characterized by three major emission bands $E_{1}, E_{2}$ and $E_{3}$ in the reported range of wavelength. These emissions correspond to the transition from ${ }^{2} H_{11 / 2} \rightarrow{ }^{4} I_{15 / 2}$, ${ }^{4} F_{9 / 2} \rightarrow{ }^{4} I_{15 / 2}$ and ${ }^{4} S_{3 / 2} \rightarrow{ }^{4} I_{15 / 2}$, respectively [15].

The symmetry of the local environment around RE ions can be studied by investigating the Stark splitting of the RE ions in a particular environment. The symmetry of such an arrangement is primarily due to the interaction of the electric potential with the orbital degeneracy of the RE ions.

3.2a Stark splitting: The energy levels of the RE ions are influenced by the surrounding environment. If such an ion is embedded in a matrix, it experiences an electric potential

Table 2. Position and width (in $\mathrm{cm}^{-1}$ ) of the Stark levels for the different transitions in Er-doped TBZ glass.

\begin{tabular}{lcc}
\hline Manifold & Position $\left(\mathrm{cm}^{-1}\right)$ & Width $\left(\mathrm{cm}^{-1}\right)$ \\
\hline${ }^{2} H_{11 / 2} \rightarrow{ }^{4} I_{15 / 2}$ & 0 & 113.13 \\
& 135.12 & 476.09 \\
& 351.05 & 352.12 \\
${ }^{4} F_{9 / 2} \rightarrow{ }^{4} I_{15 / 2}$ & 485.52 & 172.35 \\
& 0 & 226.02 \\
& 123.91 & 149.14 \\
& 242.42 & 63.68 \\
${ }^{4} S_{3 / 2} \rightarrow{ }^{4} I_{15 / 2}$ & 293.68 & 52.2 \\
& 355.09 & 281.19 \\
& 0 & 126.85 \\
& 109.74 & 178.29 \\
& 332.86 & 206.36 \\
& 448.38 & 160.54 \\
\hline
\end{tabular}

Position of the first de-convoluted peak for each transition is taken as the reference.

Table 3. Position and width (in $\mathrm{cm}^{-1}$ ) of the Stark levels for the different transitions in Eu-doped TBZ glass.

\begin{tabular}{lcc}
\hline Manifold & Position $\left(\mathrm{cm}^{-1}\right)$ & Width $\left(\mathrm{cm}^{-1}\right)$ \\
\hline${ }^{5} D_{0} \rightarrow{ }^{7} F_{1}$ & 0 & 171.35 \\
& 98.65 & 80.12 \\
${ }^{5} D_{0} \rightarrow{ }^{7} F_{2}$ & 199.82 & 136.08 \\
& 0 & 95.5 \\
& 100.76 & 139.96 \\
& 205.76 & 105.37 \\
${ }^{5} D_{0} \rightarrow{ }^{7} F_{4}$ & 289.09 & 124.67 \\
& 328.38 & 232.01 \\
& 0 & 208.62 \\
& 145.75 & 213.37 \\
\hline
\end{tabular}

Position of the first de-convoluted peak for each transition is taken as the reference. whose symmetry is same as that of the matrix responsible for the potential. Splitting of free RE ion manifold leads to Stark levels. Since a glassy host has very low symmetry, we can assume that the RE manifolds are split fully. In figure 6, we show the Stark splitting of the Eu- and Er-doped TBZ glasses. Figure $6 \mathrm{a}-\mathrm{c}$ represents the Stark splitting in Eu-doped glass for transitions ${ }^{5} D_{0} \rightarrow{ }^{7} F_{1},{ }^{5} D_{0} \rightarrow{ }^{7} F_{2}$ and ${ }^{5} D_{0} \rightarrow{ }^{7} F_{3}$, respectively. The other two transitions ( $F_{1}$ and $F_{4}$ of figure $5 a$ ) are not considered because of the sharpness and low intensity, respectively. Similarly, figure 6d-f represents the Er-doped glass for transitions ${ }^{2} H_{11 / 2} \rightarrow{ }^{4} I_{15 / 2},{ }^{4} F_{9 / 2} \rightarrow{ }^{4} I_{15 / 2}$ and ${ }^{4} S_{3 / 2} \rightarrow{ }^{4} I_{15 / 2}$, respectively. These peaks are also de-convoluted into Gaussian peaks, which represent the extent of Stark splitting of the particular transition. In $\mathrm{Eu}^{3+}$ ion, the upper state ${ }^{5} D_{0}$ is a singlet state, whereas some of the low lying ${ }^{7} F_{n}$ has multiplet nature. On the other hand, in $\mathrm{Er}^{3+}$ ion, the ground state ${ }^{4} I_{15 / 2}$ itself has manifold. Higher states like ${ }^{2} F_{9 / 2}$ and ${ }^{4} S_{3 / 2}$ also have multiplet nature. Tables 2 and 3 summarize the position and the width of Stark levels in Eu-TBZ and Er-TBZ glasses, respectively, for the above said transitions. Position of the each peak defines the particular Stark component and its FWHM is a measure of the inhomogeneous broadening [16].

The broadening of the Stark levels can be attributed to two origins: (a) homogeneous broadening which is related to a given ion in the matrix and (b) inhomogeneous broadening which is global distribution of all the homogeneous contributions.

Homogeneous broadening is because of many contributions, viz., direct processes, Raman and Orbach processes, multiphonon and radiative relaxations. These processes lead to a Lorenzian line shape.

Inhomogeneous broadening in case of glassy matrix is related to the distribution of different sites for the RE ion. It is assumed that the broadening results in a Gaussian line shape. From the PL data, one can estimate this broadening

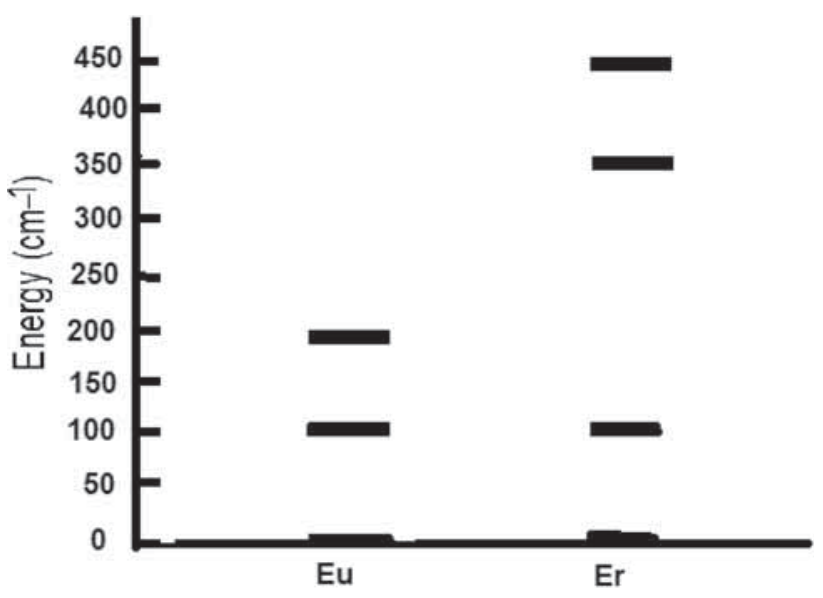

Figure 7. Comparison of the Stark splitting of the transition ${ }^{5} D_{0} \rightarrow{ }^{7} F_{1}$ in Eu-doped and ${ }^{4} S_{3 / 2} \rightarrow{ }^{4} I_{15 / 2}$ transition in Er-doped TBZ glasses. 

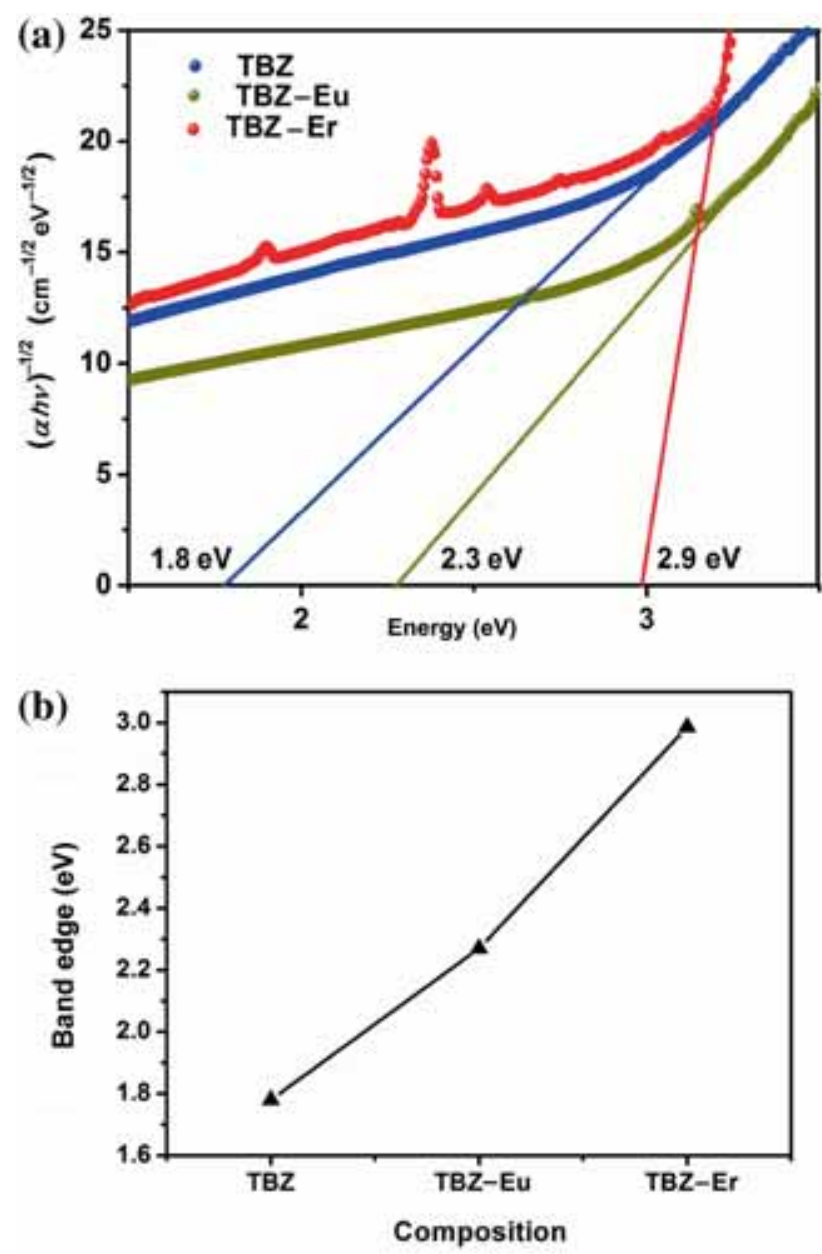

Figure 8. (a) Plot of $(\alpha h v)^{1 / n} v s$. (hv) according to equation (1) to estimate the optical band edge. The estimated band edge is mentioned at the bottom of each extrapolated line. (b) Band edge against the undoped and RE-doped glasses.

by fitting Gaussian line shape to different Stark levels and finding out the FWHM of each Stark splitting.

Tables 2 and 3 show the position of Stark levels and the extent of inhomogeneous broadening of the selected transitions in Er-doped and Eu-doped glasses, respectively. From the tables, it is observed that the broadening is smaller in Eu-doped glass suggesting a more uniform environment [17]. From the energy level diagram of figure $5 \mathrm{c}$, we observe that the transitions ${ }^{5} D_{0} \rightarrow{ }^{7} F_{1}$ of Eu-doped glass and ${ }^{4} S_{3 / 2} \rightarrow{ }^{4} I_{15 / 2}$ of Er-doped glass are energetically almost equivalent. In figure 7, Strark levels of these transitions are plotted. It can be seen that the Stark levels of Eu-doped TBZ glass are equally spaced, whereas for Er-doped glass, the splitting is larger. The larger Strark splitting in case of Er-doped TBZ glass shows the presence of a higher crystal-field. The two Stark levels which are higher shows that the symmetry assumed may not be as low as usually assumed for glass $\left(\mathrm{C}_{2 \mathrm{v}}\right)$ [18]. The fact that the Stark levels are equally spaced in Eu-doped TBZ glasses than that of the Er-doped, one can infer that all ions in Eu-doped glass are located in similar sites, having small deformations from each other [17].

\subsection{UV-visible absorption}

Generally, the UV-vis spectra are analysed using Mott and Davis [19] formula for the absorption coefficient:

$$
\alpha(v)=\frac{A\left(h v-E_{\mathrm{g}}\right)^{n}}{h v}
$$

where $E_{\mathrm{g}}$ is the value of optical bandgap (band edge) and $n$ is the exponent which characterizes the direct or indirect mode of electronic transitions during the absorption process in the $\mathrm{k}$-space. The exponent $n$ can possess the values of $0.5,1.5$, 2 and 3 for direct allowed, direct forbidden, indirect allowed and indirect forbidden, respectively. The factor $A$ is a constant within the frequency range which depends on the transition probability.

The usual method for the determination of the value of $E_{\mathrm{g}}$ involves plotting $(\alpha h \nu)^{1 / n}$ against $(h \nu)$. We have tried to fit equation (1) for all the values of $n$ mentioned above, but the most satisfactory results were obtained using $n=0.5$ as shown in figure 8a. This shows that glasses under the study are characterized by direct bandgap. Figure $8 \mathrm{~b}$ shows the optical bandgap estimated using the above equation $v s$. the RE ions. Eu-doped TBZ glass shows a lesser bandgap than Er-doped one.

Europium has a larger ionic radius than Er. Insertion of Eu into the glass matrix depolymerizes the matrix, leading to a more homogeneous environment. This is observed from the Raman peak shift and equal spacing of the Stark splitting. Homogeneous environment leads to lower bandgap [20].

\section{Conclusions}

From the peak shift, intensity variation and FWHM of the Raman spectra of the glass samples, it is observed that Eu-doped TBZ glass has a greater tendency towards depolymerizing the glass matrix by influencing conversion of $\mathrm{TeO}_{4}$ units into $\mathrm{TeO}_{3}$ units. PL spectra of the glass samples show emission due to different possible transitions. Position of the peak of the de-convoluted spectra shows the position of the particular Stark component and the FWHM is a measure of the inhomogeneous broadening. From the analysis of Stark components and the inhomogeneous broadening, it is observed that Eu-doped TBZ glass has more uniform environment than the Er-doped glass. Er-doped glasses are at a higher symmetry than the usual $\mathrm{C}_{2 \mathrm{v}}$ symmetry assumed for the glass. From all these, it can be concluded that Eu ion is in a more uniform environment than the Er ion in the given glass matrix. The UV-visible absorption spectra are used to calculate the optical density and fitted to the Mott-Davis equation to determine the band edge of the glass samples. It is seen that Eu-doped 
TBZ glass has a lesser bandgap than that of Er-doped glass. All the glasses are characterized by the presence of direct bandgap.

\section{Acknowledgements}

One of the authors $(\mathrm{GVH})$ would like to thank the management of the PES for sponsoring and the AICTE for granting QIP fellowship.

\section{References}

[1] Righini G C and Ferrari M 2005 Riv. del Nuovo Cim. 281

[2] Judd B R 1962 Phys. Rev. 127750

[3] Ofelt G S 1962 J. Chem. Phys. 37511

[4] Binnemans K and Görller-Walrand C 1998 J. Phys. Condens. Matter 10 L167

[5] Mukhopadyay S $2005 \mathrm{PhD}$ Thesis, Department of Physics, Indian Institute of Science, Bangalore, India

[6] Wang J S, Vogel E M and Snitzer E 1994 Opt. Mater. (Amst) 3 187

[7] Simmons C J and Simmons J H 1986 J. Am. Ceram. Soc. 69 661
[8] Moynihan C T and Loehr S R 1991 Mater. Sci. Forum 32-33 243

[9] Binnemans K, Van Deun R, Görller-Walrand C and Adam J L 1998 J. Non-Cryst. Solids 23811

[10] Sekiya T, Mochida N and Ohtsuka A 1994 J. Non-Cryst. Solids 168106

[11] Manning S, Ebendorff-Heidepriem H and Monro T M 2012 Opt. Mater. Exp. 2140

[12] Sidebottom D L and Hruschka M A, Potter B G and Brow R K 1997 Appl. Phys. Lett. 711963

[13] Honnavar G V, Prabhava S N and Ramesh K P 2013 J. NonCryst. Solids 3706

[14] Babu P, Jang K H, Seo H J and Jayasankar C K 2006 J. Appl. Phys. 99053522

[15] Vetrone F, Boyer J-C, Capobianco J A, Speghini A and Bettinelli M 2002 Appl. Phys. Lett. 801752

[16] Francini R, Grassano U M and Tarasov G G 2001 J. Chem. Phys. 1157975

[17] Bigot L, Jurdyc A-M, Jacquier B, Gasca L and Bayart D 2002 Phys. Rev. B 66214204

[18] Mortier M, Huang Y D and Auzel F 2000 J. Alloys Compd. 300-301 407

[19] Mott N F and Davis E A 1971 Electronic process in noncrystalline materials (UK: Oxford University Press)

[20] Zanatta A R and Chambouleyron I 1996 Phys. Rev. B 53 3833 